\title{
Tratamiento de la pancreatitis crónica mediante el procedimiento de DuVal laparoscópico: reporte de un caso pediátrico
}

\author{
Treatment of chronic pancreatitis by laparoscopic DuVal procedure: report of a pediatric case
}

Carlos García-Hernández*, Lourdes Carvajal-Figueroa, Cristian Archivaldo-García y Sergio Landa-Juárez Departamento de Cirugía Pediátrica, Hospital Infantil Privado, Curso de Alta Especialidad Postgrado, Universidad Autónoma de México, Ciudad de México, México

\section{Resumen}

Antecedentes: La pancreatitis es una enfermedad rara. La obstrucción es común y se puede corregir con endoscopia; si fracasa, necesitará cirugía. Caso clínico: Reportamos un paciente con pancreatitis secundaria a páncreas divisum. Se realizó derivación tipo DuVal laparoscópica. Varón de 12 años, con múltiples cuadros de pancreatitis y fallidos intentos de esfinteroplastias. La colangiorresonancia mostró páncreas divisum. Se realizó una derivación tipo DuVal por laparoscopia. Seguimiento de 5 años. No ha habido recurrencia del dolor y la lipasa se normalizó a los 6 meses. El conducto pancreático no estaba dilatado. Ante la dificultad para hacer una anastomosis lateral se utilizó la técnica laparoscópica propuesta por DuVal.

Palabras clave: Pancreatitis crónica. Procedimiento de DuVal. Laparoscopia.

\begin{abstract}
Background: Pancreatitis is rare. Obstruction is common. They can be corrected with endoscopy, if they fail they need surgery. Case report: We reported a patient with pancreatitis secondary to pancreas divisum, laparoscopic DuVal shunt was performed. Male 12 years, multiple pancreatitis pictures and failed sphinteroplasty attempts. Colangio resonance showed pancreas divisum. A DuVal-type shunt was built for laparoscopy. Follow-up 5 years. There has been no recurrence of pain and lipase was normalized at 6 months. The pancreatic duct was not dilated, in the face of difficulty making a lateral anastomosis was used the technique proposed by DuVal by laparoscopic approach.
\end{abstract}

Key words: Chronic pancreatitis. DuVal procedure. Laparoscopy.

\section{Introducción}

La pancreatitis es una enfermedad relativamente rara en pediatría; su incidencia se calcula en 1 por cada 50,000 niños ${ }^{1}$. En la forma crónica, el parénquima pancreático es destruido y sustituido por tejido fibroso². Se caracteriza por episodios recurrentes de dolor intenso y otros síntomas como diarrea, esteatorrea y diabetes mellitus, síntomas secundarios a insuficiencia exocrina y endocrina ${ }^{3}$. La etiología es variada, pero las

Correspondencia:

*Carlos García-Hernández

Circuito Valle Dorado, 12

Col. Loma de Valle Escondido

Fecha de recepción: 06-05-2020

C.P. 52930, Atizapán de Zaragoza, Edo. de México, México

E-mail: carloscirped@hotmail.com
Cir Cir. 2021;89(2):258-262

Contents available at PubMed

www.cirugiaycirujanos.com (http://creativecommons.org/licenses/by-nc-nd/4.0/). 
causas obstructivas debido a alteraciones del conducto pancreático son las más comunes ${ }^{1,4,5}$. Esta obstrucción al flujo pancreático aumenta la presión del conducto y del parénquima, lo que produce el dolor abdominal característico ${ }^{6}$. Este síntoma es la indicación más común de tratamiento quirúrgico ${ }^{7}$, cuyos objetivos son eliminar el dolor y preservar la función del páncreas. En los casos con alteraciones en el drenaje pancreático, este se puede corregir con métodos endoscópicos, y si fracasan, se necesitará algún tipo de tratamiento quirúrgico, por lo que es importante conocer la anatomía del conducto pancreático ${ }^{8}$. Del $5 \%$ al $20 \%$ de los pacientes con pancreatitis tienen anomalías anatómicas, siendo la más común el páncreas divisum ${ }^{9}$. El páncreas divisum es una anomalía embrionaria de los conductos pancreáticos debida a una falta de fusión de los conductos dorsal y ventral del páncreas ${ }^{10}$, y fue descrito por Opie en $1903^{11}$. A pesar de ser controvertida la relación con el desarrollo de pancreatitis, existen diversos reportes de su asociación con esta enfermedad ${ }^{12-15}$. Cuando un paciente cursa con pancreatitis sin evidencia de otra etiología y con el hallazgo de páncreas divisum, se le deberá considerar como causante de este cuadro y se tratará para evitar complicaciones ${ }^{10}$. El primer reporte de un abordaje laparoscópico en adultos para construir una derivación hacia el yeyuno fue publicado en $2004^{16}$. El primer reporte en niños de la construcción de una derivación pancreaticoyeyunal lateral por robótica fue publicado en $2011^{17}$. Han aparecido solo algunos reportes de abordajes laparoscópicos con la construcción de una anastomosis pancreaticoyeyunal lateral por mínima invasión en niños, que tienen en común el presentar una dilatación del conducto pancreático ${ }^{18,19}$. Para la construcción de este tipo de derivaciones pancreaticoyeyunales laterales tipo Puestow se requiere que exista dilatación del conducto pancreático ${ }^{19-21}$. En 1954, Merlin K. DuVal propuso realizar una anastomosis pancreaticoyeyunal terminolateral para descomprimir el páncreas que no requiere dilatación del conducto pancreático ${ }^{22}$.

El objetivo de este trabajo es reportar el caso de un paciente pediátrico con pancreatitis crónica secundaria a páncreas divisum, sin dilatación del conducto pancreático, con falla del tratamiento endoscópico, a quien se realizó una derivación pancreaticoyeyunal tipo DuVal laparoscópica tradicional con control del proceso inflamatorio pancreático.

\section{Caso clínico}

Varón de 12 años, con antecedente de autismo, el cual inició su padecimiento a los 3 años, con un cuadro de abdomen agudo que se documentó, mediante laparotomía exploradora con diagnóstico preoperatorio de apendicitis aguda, como un cuadro abdominal secundario a pancreatitis aguda. Debido a una evolución tórpida, a los 10 días se practicó una colecistectomía abierta. A partir de este evento, el paciente presentó de tres a cuatro episodios por año de pancreatitis aguda, caracterizados por dolor intenso, incapacitante, con elevación de las enzimas pancreáticas, que requirieron hospitalización, manejo con narcóticos y ayuno, con remisión temporal de los síntomas. A los 8, 9 y 11 años de edad, se llevaron a cabo tres intentos fallidos de esfinteroplastia endoscópica con colocación de stents al conducto pancreático. A los 12 años de edad presentó un nuevo cuadro de pancreatitis, con elevación de la amilasa y la lipasa séricas. Se practicó una colangiopancreatografía mediante resonancia magnética, simple en $\mathrm{T}$ 2, que mostró una división parcial del conducto pancreático principal compatible con un páncreas divisum incompleto, con disminución del calibre de los conductos de Wirsung y Santorini, y con un conducto pancreático de $5 \mathrm{~mm}$ (Fig. 1). Debido a esta nueva recaída, sin haber podido colocar stents en el conducto pancreático y ante la evidencia de esta anomalía anatómica, se construyó una derivación del páncreas distal al yeyuno, con resección de la cola del páncreas y anastomosis terminal al yeyuno, tipo DuVal, por laparoscopia. La técnica quirúrgica fue la siguiente: paciente en decúbito dorsal, colocación del cirujano entre las piernas del paciente, camarógrafo a la derecha y asistente a la izquierda del paciente; abordaje de cuatro puertos (uno de $10 \mathrm{~mm}$ transumbilical, uno de $3 \mathrm{~mm}$ en la línea media clavicular derecha, otro de $5 \mathrm{~mm}$ en la línea media clavicular izquierda y otro más de $3 \mathrm{~mm}$ en la línea axilar anterior izquierda); óptica de $5 \mathrm{~mm}$ y $30^{\circ}$, con una presión de $10 \mathrm{mmHg}$ e insuflación de 1 l/min; elevación gástrica con punto percutáneo, disección del epiplón hasta ver el páncreas en su totalidad, disección de la cola del páncreas separándola de los vasos esplénicos, hasta poder luxarla y separarla del cuerpo del páncreas (Fig. 2); a $20 \mathrm{~cm}$ del ángulo de Treitz se cortó y separó el intestino, utilizando una grapadora Endo GIA (Covidien Company, Minneapolis, USA) con un cartucho de $45 \mathrm{~mm}$, que se reforzó con un segundo plano con sutura continua de seda 2-0; se ascendió el asa de yeyuno distal a través del mesenterio del colon y se resecó la cola del páncreas, incidiendo la periferia del páncreas con bisturí armónico y la parte central con tijera para evitar dañar el 


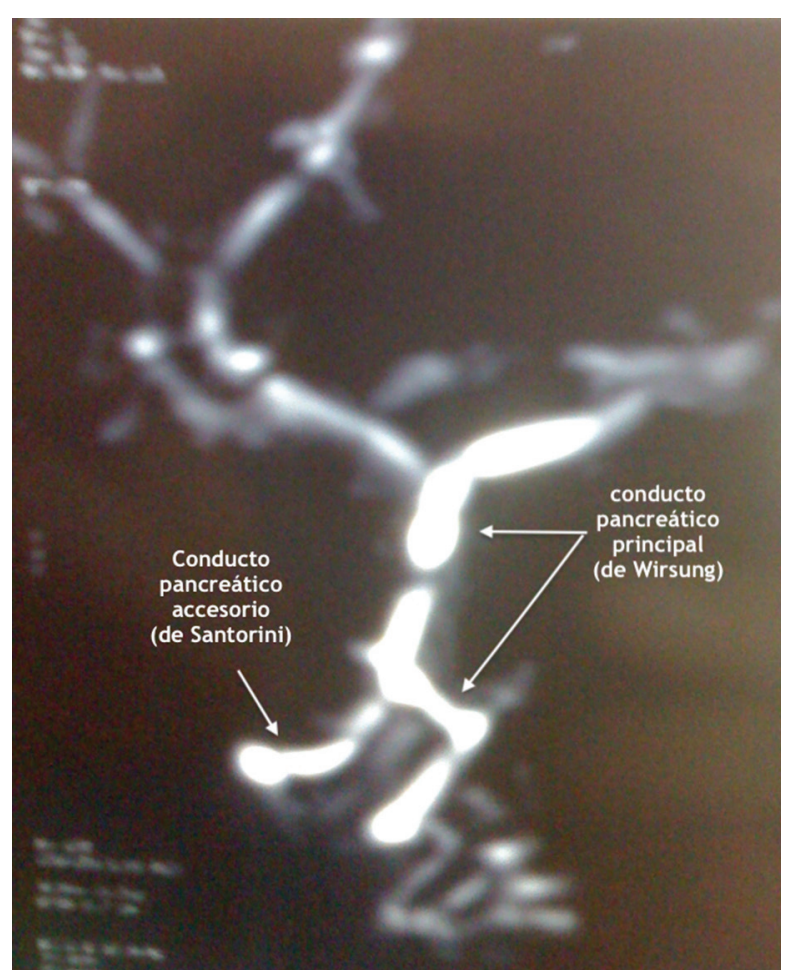

Figura 1. Colangiorresonancia magnética, simple en $T 2$, en la que se observa una división parcial del conducto pancreático principal, compatible con un páncreas divisum incompleto, con disminución del calibre de los conductos de Wirsung y Santorini, y con un conducto pancreático de $5 \mathrm{~mm}$.

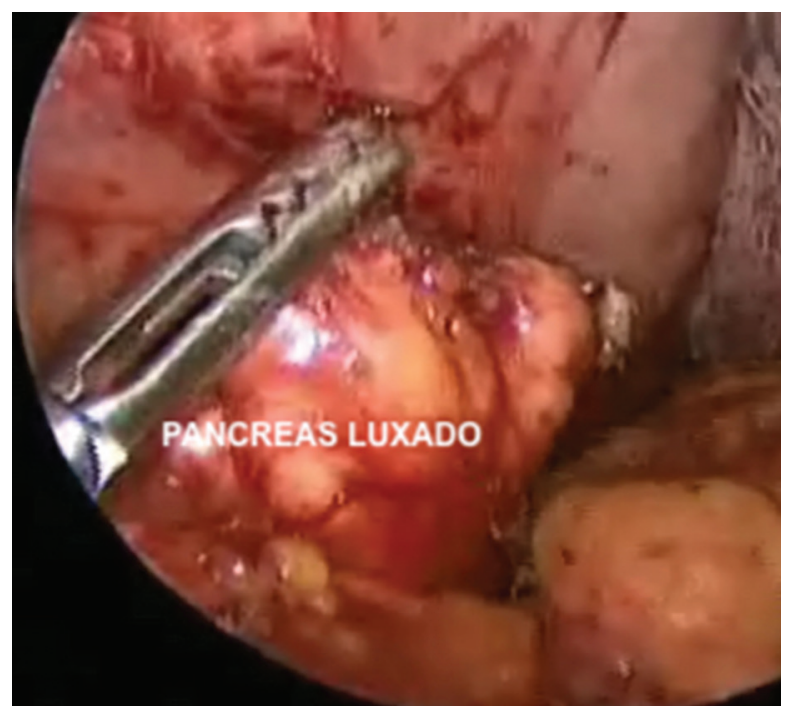

Figura 2. Cola del páncreas luxada, respetando los vasos esplénicos.

conducto pancreático (Fig. 3); se incidió el borde antimesentérico del asa de yeyuno ascendida y se practicó una anastomosis terminolateral con seda 2-0 en un plano con surgete continuo (Fig. 4); y a

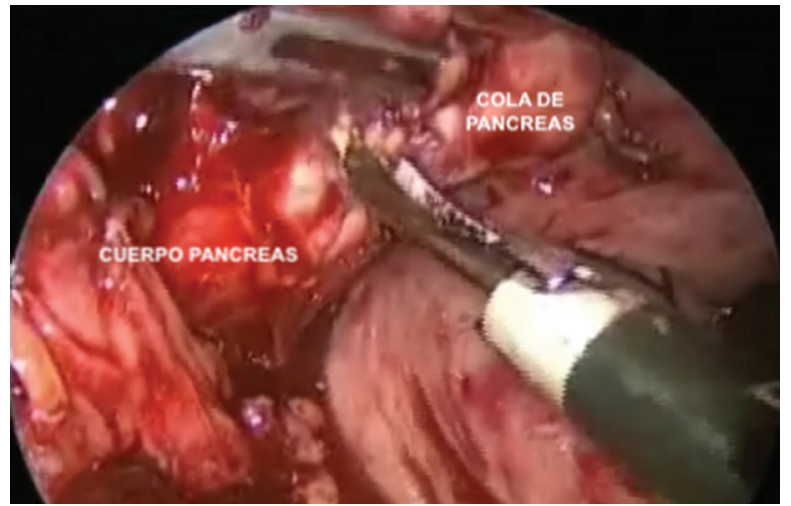

Figura 3. Resección de la cola de páncreas con bisturí armónico.

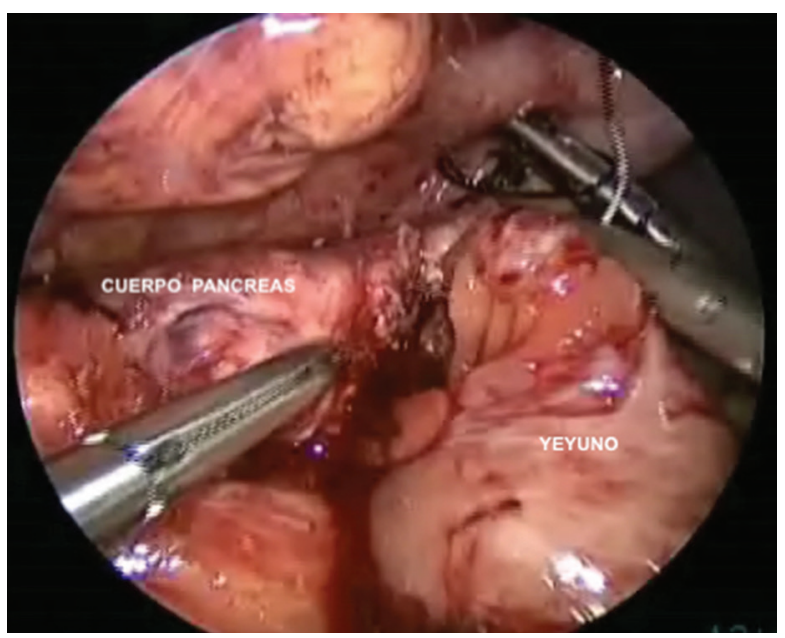

Figura 4. Anastomosis en un plano del cuerpo del páncreas al asa de yeyuno.

continuación se llevó a cabo de manera videoasistida la anastomosis terminolateral en $Y$ de Roux en un plano con sutura no absorbible 3-0.

El tiempo quirúrgico fue de 163 minutos. El paciente cursó sin complicaciones, se inició la vía oral al quinto día y se egresó al séptimo día. La cifra de lipasa descendió de 1210 a $400 \mathrm{U} / \mathrm{l}$ al momento del egreso. El seguimiento fue clínico y laboratorial a 5 años, evaluando la presencia de dolor abdominal y con determinaciones de la amilasa y la lipasa séricas. No ha habido recurrencia del dolor y la lipasa se normalizó a los 6 meses del posoperatorio, manteniéndose en rango normal hasta 5 años después de la operación, con determinaciones semestrales (Fig. 5). La actividad pancreática se valoró con la función endocrina, con cifras de glucemia normales, y la exocrina con determinación de la actividad tríptica y de grasas en heces, 




Figura 5. Cuantificación posoperatoria de la lipasa sérica, con cifras normales a partir de la semana 5 de posoperatorio y sin elevaciones que indiquen una recaída en un seguimiento de 5 años (normal: 23-300 U/I).



Figura 6. Imagen histopatológica que muestra atrofia de lobulillos con desaparición de los islotes de Langerhans; hallazgos compatibles con pancreatitis crónica.

normales a 5 años. Debido a la buena evolución clínica y de laboratorio, no se consideró necesario realizar ningún estudio radiológico. El reporte de patología del segmento de páncreas resecado fue de atrofia de lobulillos con desaparición de los islotes de Langerhans, con contenido proteínico en su lumen, con ectasia ductal y fibrosis intersticial moderada, hallazgos compatibles con pancreatitis crónica (Fig. 6).

\section{Discusión}

El tratamiento de la pancreatitis crónica va a depender de la etiología. La morfología de los conductos pancreáticos debe ser conocida mediante colangiopancreatografía retrógrada endoscópica o colangiopancreatografía con resonancia magnética. Hasta en el $90 \%$ de los niños es posible realizar una colangiopancreatografía retrógrada endoscópica, la cual, aparte de ser diagnóstica, puede ser también terapéutica ${ }^{8}$. La colangiopancreatografía realizada mediante resonancia magnética visualiza ambos conductos $^{11}$, lo que permite valorar el conducto pancreático para poder planear el tipo de tratamiento. Si bien hay controversia acerca del papel del páncreas divisum como causa de pancreatitis crónica, existen diversos reportes de que el tratamiento de esta malformación permite el control del cuadro inflamatorio crónico ${ }^{10,12-15}$, tal como sucedió en el caso que presentamos. Nuestro paciente tenía las siguientes indicaciones de tratamiento quirúrgico: dolor intratable, alteraciones en el drenaje pancreático y fracaso de los procedimientos endoscópicos ${ }^{7}$. Existen diferentes opciones de tratamiento quirúrgico, cada una con sus ventajas y desventajas, por lo que el tipo de tratamiento se debe individualizar para cada pacien$t^{3,18}$. En pediatría existen publicaciones con las técnicas de Frey o Puestow llevadas a cabo con cirugía tradicional ${ }^{19,20}$. El procedimiento que se ha realizado con mayor frecuencia es con la técnica de Puestow, que requiere que el conducto esté dilatado para poder realizar una anastomosis pancreaticoyeyunal lateral. En nuestro paciente, el conducto pancreático no estaba dilatado, por lo que se había planteado llevar a cabo una pancreatectomía total para el control de los síntomas; sin embargo, considerando que realizar este procedimiento, aun con trasplante de islotes pancreáticos, tiene una posibilidad de hasta el $58.7 \%$ de requerir insulina, además de cursar con insuficiencia exocrina de vida ${ }^{21,22}$, en un paciente con autismo y ante la dificultad técnica para localizar el conducto, que no se encontraba dilatado, lo que dificultaría una anastomosis lateral al yeyuno, decidimos utilizar la técnica propuesta por DuVal ${ }^{23}$. Se realizó una resección de la cola del páncreas hasta el cuerpo, el mismo con anastomosis terminal del páncreas hacia el yeyuno, con preservación de la circulación esplénica, que fue posible llevarla a cabo mediante laparoscopia. Esta técnica tiene también la ventaja de resecar una zona en la que se espera que haya fibrosis, como demostró el reporte histopatológico, además de que es posible confirmar la permeabilidad de la derivación yeyunal mediante colangiopancreatografía retrógrada endoscópica varios años después de la cirugía ${ }^{23}$. Son muy escasos los reportes de estos procedimientos en niños por abordajes 
mínimamente invasivos. En este caso fue posible el abordaje laparoscópico, a pesar de las operaciones previas, lo que permitió una adecuada disección de los vasos esplénicos y del páncreas, permitiendo la construcción de un drenaje hacia el yeyuno funcional, sin fugas ni fistulas, con preservación del bazo.

Esperamos a tener un seguimiento a 5 años para contar con la certeza de que existe un control de los procesos inflamatorios del páncreas. Además, en caso de recidiva, existe siempre la opción de realizar algún otro procedimiento, como el de Puestow o el de Frey ${ }^{24-26}$, antes de considerar la opción de la resección completa del páncreas en un niño con autismo.

El páncreas divisum es una causa importante de pancreatitis recurrente en niños y debe ser identificada en los pacientes que tengan más de un episodio de pancreatitis ${ }^{15,16}$. El tratamiento quirúrgico está dirigido a aliviar la obstrucción del conducto pancreático; cuando esto no es posible mediante la colocación de un stent, como sucedió en nuestro paciente, se deberá llevar a cabo una derivación del páncreas al yeyuno ${ }^{10,14}$. Cuando exista dilatación del conducto pancreático se puede realizar una derivación tipo Puestow ${ }^{20}$; sin embargo, si el conducto no está dilatado, el abordaje tipo DuVal es una buena opción ${ }^{22}$.

En este paciente fue factible llevar a cabo este procedimiento de derivación pancreaticoyeyunal mediante cirugía de mínima invasión, sin incidentes, con un adecuado control de la enfermedad a largo plazo y conservando las ventajas ya conocidas de la laparoscopia. Sin embargo, es necesario un mayor número de casos para validar el procedimiento de DuVal laparoscópico.

\section{Financiamiento}

No se recibió ningún financiamiento para elaborar este trabajo.

\section{Conflicto de intereses}

Los autores no tienen ningún conflicto de intereses que declarar.

\section{Responsabilidades éticas}

Protección de personas y animales. Los autores declaran que los procedimientos seguidos se conformaron a las normas éticas del comité de experimentación humana responsable y de acuerdo con la Asociación Médica Mundial y la Declaración de Helsinki.
Confidencialidad de los datos. Los autores declaran que han seguido los protocolos de su centro de trabajo sobre la publicación de datos de pacientes.

Derecho a la privacidad y consentimiento informado. Los autores han obtenido el consentimiento informado de los pacientes y/o sujetos referidos en el artículo. Este documento obra en poder del autor de correspondencia.

\section{Bibliografía}

1. Clifton MS, Pelayo JC, Cortes RA, Grethel EJ, Wagner AJ, Lee H, et al. Surgical treatment of childhood recurrent pancreatitis. J Pediatr Surg. 2007;42:1203-7.

2. Bachmann K, Mann O, Izbicki JR, Strate T. Chronic pancreatitis - a surgeon's view. Med Sci Monit. 2008;14:RA198-205.

3. Anderson DK, Frey CF. The evolution of the surgical treatment of chronic pancreatitis. Ann Surg. 2010;251:18-32.

4. Weber TR, Keeller M. Operative management of chronic pancreatitis in children. Arch Surg. 2001;136:550-5.

5. O'Neill Jr JA, Greene H, Grishan FK. Surgical implications of chronic pancreatitis. J Pediatr Surg. 1982;17:920-6.

6. Demir IE, Tieftrunk E, Maak M, Friess H, Ceyhan GO. Pain mechanisms in chronic pancreatitis: of a master and his fire. Langenbecks Arch Surg. 2011;396:151-60.

7. Aimoto T, Uchida E, Nakamura Y, Yamahatsu K, Matsushita A, Katsuno A, et al. Current surgical treatment for chronic pancreatitis. $J$ Nippon Med Sch. 2011;78:352-9.

8. Allendorph M, Werlin SL, Greenen JE, Hogan WJ, Venu RP, Stewart ET, et al. Endoscopic retrograde cholangiopancreatography in children. $J$ Pediatr. 1987;110:206-11.

9. Uc A, Husain SZ. Pancreatitis in children. Gastroenterology. 2019;156:1969-78.

10. Neblett WW, O'Neill J. Surgical management of recurrent pancreatitis in children with pancreas divisum. Ann Surg. 2000;231:899-908.

11. Opie E. The anatomy of the pancreas. Johns Hopkins Hospital Bull. 1903;14:229-32.

12. Mitchelll CJ, Lintott DJ, Ruddell WSJ, Losowsky MS, Axon AT. Clinical relevance of an unfused pancreatic duct system. Gut. 1979;20:1066-71.

13. Delhaye M, Engelholm L, Cremer M. Pancreas divisum: congenital anatomic variant or anomaly? Contribution of endoscopic retrograde dorsal pancreatography. Gastroenterology. 1985;89:951-8.

14. Richter JM, Shapiro RH, Mulley AG, Warshaw AL. Association of pancreas divisum and pancreatitis, and its treatment by sphinteroplasty of the accesory ampulla. Gastroenterology. 1981;81:1104-10.

15. Warshaw AL, Simeone JF, Scahpiro RH, Flavin-Warshaw B. Evaluation and treatment of the dominant dorsal duct syndrome (pancreas divisum redefined). Am J Surg. 1990;159:59-64.

16. Glaser C, Mülleer W, Zerz A, Szinicz G. [Laparoscopic latero-lateral pancreaticojejunostomy]. Chirurg. 2000;71:456-7.

17. Meehan JJ, Sawn R. Robotic lateral pancreaticojejunostomy (Puestow). J Pediatr Surg. 2011;64:E5-E8.

18. Arora A, Agarwal P, Bagdi P, Ramasundaram M, Sankar Narayanan ML. Laparoscopic Puestow procedure for chronic pancreatitis in children. $J$ Indian Pediatr Surg. 2020;25:55-7.

19. Shah AA, Petrosyan M, Kane TD. Lateral pancreaticojejunostomy for chronic pancreatitis and pancreatic ductal dilation in children. J Lapaparoendosc Adv Surg Tech A. 2018;11:1397-402.

20. Puestow CB, Gillesby WJ. Retrograde surgical drainage of pancreas for chronic relapsing pancreatitis. AMA Arch Surg. 1958;76:898-907.

21. Chinnakotla S, Bellin MD, Schwarzenberg SJ, Radosevich DM, Cook M, Dunn TB, et al. Total pancreatectomy and islet auto-transplantation in children for chronic pancreatitis. Indication, surgical techniques, postoperative management and long-term outcomes. Ann Surg. 2014;260: 56-64.

22. Kai Bachmann K, Kutup A, Mann O, Yekebas E, Izbicki JR. Surgical treatment in chronic pancreatitis timing and type of procedure. Best Pract Res Clin Gastroenterol. 2010;24:299-310.

23. DuVal MK. Caudal pancreatico-jejunostomy for chronic relapsing pancreatitis. Ann Surg. 1954;140:775-85.

24. Rollins MD, Meyers RL. Frey procedure for surgical management of chronic pancreatitis in children. J Pediatr Surg. 2004;39:817-820.

25. DuBay D, Sandler A, Kimura K, Bishop W, Eimen M, Soper R. The modified Puestow procedure for complicated hereditary pancreatitis in children. J Pediatr Surg. 2000;35:343-8.

26. Weber TR, Keller MS. Operative management of chronic pancreatitis in children. Arch Surg. 2001;136:550-5. 